DOI: $10.15240 /$ tul/004/2020-3-004

\title{
AUSGEWÄHLTE WESTSCHLESISCHE SCHLOSSRESIDENZEN ALS KULTURELLE REPRÄSENTANZEN ZWISCHEN BAROCK UND AUFKLÄRUNG. ABRISS DER PROBLEMATIK IM LITERATUR- UND KULTURHISTORISCHEN BLICKWINKEL
}

\author{
Iveta Zlá \\ Universität Ostrava, Philosophische Fakultät, Lehrstuhl für Germanistik, \\ Reální 5, 70100 Ostrava, Česká republika \\ e-mail: iveta.zla@osu.cz
}

\begin{abstract}
Die Studie konzentriert sich auf die Darstellung eines vielfältigen kulturellen Bildes, von dem sich das künstlerische Leben in Westschlesien zwischen Barock und Aufklärung nicht trennen lässt. Obwohl die Zeit zwischen Barock und Aufklärung in einigen Teilen Schlesiens einen Aufschwung verzeichnet hat, steht im Vordergrund der Untersuchung das kulturelle Leben in den Dominien Rosswald, Groß Hoschütz und Johannesberg.
\end{abstract}

\section{Keywords}

West Silesia; Rosswald; Großhoschütz; Johannesberg; Culture; Theatre; Music; Ballet; Cultural cooperation.

\section{Einführung}

Westschlesien erfreute sich in der Zeit zwischen Barock und Aufklärung einer enormen Kunstentwicklung. Zur künstlerischen Blüte dieses Teils Schlesiens hat vor allem das kulturelle Engagement des Grafen Albert Joseph Hoditz (1706-1778), des Bischofs Philipp Gotthard Schaffgotsch (1715-1795) sowie des Grafen Dominik Ignaz Chorinsky (1729 1792) beigetragen, zu deren Sitzen die Schlösser in Rosswald ${ }^{1}$, Johannesberg ${ }^{2}$ und Großhoschütz ${ }^{3}$ gehörten. Zwischen den genannten Schlossresidenzen bestand ein reger Gedankenaustauch, der zur Herausbildung eines einzigartigen, diesen Winkel Schlesiens weit übersteigenden, kulturellen Mosaiks beigetragen hat. Obwohl alle drei angeführten Schlossresidenzen zu den kulturellen Mittelpunkten Westschlesiens gehörten, wurde ihrer kulturellen Präsenz sowie dem künstlerischen Gedankenaustausch bisher relativ wenig Aufmerksamkeit [12] geschenkt. Die vorliegende Studie bemüht sich, dieses Defizit zu beseitigen.

\section{$1 \quad$ Ziele der Untersuchung}

Die Grundintentionen der Untersuchung sind mit dem Einblick in das kulturelle Bild der angeführten Schlösser verbunden. Im Mittelpunkt stehen die Skizzierung der künstlerischen Atmosphäre in diesen Kunststätten sowie der Einblick in das Repertoire des Rosswalder und Großhoschützer Schlosstheaters. Da die musikalische Tätigkeit Karl Ditters von Dittersdorfs im westschlesischen kulturellen Kolorit eine Schlüsselrolle gespielt hat, findet der Beitrag dieses Komponisten für das dortige kulturelle Leben Erwähnung.

\footnotetext{
${ }^{1}$ Das Schloss Slezské Rudoltice (Rosswald) war Sitz des Grafen Albert Joseph Hoditz.

${ }^{2}$ Das Schloss Jánský Vrch v Javorníku (Johannesberg in Jauernig) war Sitz der Breslauer Bischöfe.

${ }^{3}$ Das Schloss Velké Hoštice (Großhoschütz) war im Besitz des Grafen Dominik Ignaz Chorinsky.
} 
Methodologischer Hintergrund der Studie

Die Studie fußt auf hermeneutischen Forschungsprinzipien. Sie fokussiert nicht nur das kulturelle Leben in den ausgewählten westschlesischen Schlossresidenzen zwischen Barock und Aufklärung, sondern ist auch mit der Intention verbunden, die aus der Sichtung der Archivalien sowie aus dem Studium der Forschungsliteratur hervorgehenden Informationen in die literatur- und kulturhistorischen Zusammenhänge zu setzen.

\section{Das Schloss des Grafen Albert Joseph Hoditz als ,Sitz der Museen und Grazien"“}

Das kulturelle Renommee des Rosswalder Schlosses war mit zahlreichen Festivitäten sowie Dramen- und Ballettaufführungen verbunden. Das Schloss war von einer Gartenanlage umgeben, in der sich zahlreiche Statuen und andere künstlerische Artefakten befanden, die meistens durch die antike Mythologie inspiriert worden waren.[22] In diese Gartenlandschaft wurden die bukolisch geprägten Dramenaufführungen situiert, um die künstlerische Atmosphäre dieses Ortes zu unterstreichen. Der Graf Albert Joseph Hoditz unterstützte die Verbreitung der Kunst und Bildung und seine kulturelle Tätigkeit wurde sowohl von barocken kulturellen Impulsen beeinflusst, als auch durch die Gedanken der Aufklärung gekennzeichnet. Obwohl in Schlesien zwischen 1756 und 1763 der Siebenjährige Krieg tobte, kam es hier gerade in dieser Zeit zu einem enormen kulturellen Aufschwung.

Dem phänomenalen kulturellen Ruf des Rosswalder Schlosses entsprachen sowohl seine Attribute wie „schlesisches Arkadien“ [6], „Heiligthum der Musen und Gratien“ [6], „Feensitz“ [16] und „Schlesisches Versailles“, als auch die Charakteristiken des Grafen Albert Joseph Hoditz, die zwischen negativen und höchst positiven Bezeichnungen schwanken. Richard Trampler bezeichnete den Grafen Hoditz als einen „Sonderling in Rosswald“ [16], Johannes Richter balanciert in seinem Wortspiel zwischen ,einem närrischen Genie und einem genialen Grafen" [13], das er jedoch in seiner Monografie über diesen Adligen mit dem Wort „Der Wundergraf“ [13] ebenso wie Paul Drechsler [8] umrahmt. Die Lebensweise des Grafen Hoditz wurde bereits zu seinen Lebzeiten von Friedrich II. als epikureisch ${ }^{4}$ bezeichnet.

Das dramatische und musikalische Engagement des Grafen Albert Joseph Hoditz wurde nicht nur durch die barocke Intention der angemessenen Repräsentation begleitet, sondern auch durch seine künstlerischen Interessen und Begabungen untermauert. Die kulturellen Initiativen dieses Adligen übertreffen die mit dem aristokratischen Statut verbundenen barocken gesellschaftlichen Konventionen und münden in die Aufklärung.

Die Darlegung des Rosswalder kulturellen Panoramas wird von einem Einblick in die Büchersammlung des Grafen Hoditz begleitet, ${ }^{5}$ der nicht nur den literarischen Geschmack des Grafen Albert Joseph Hoditz und seinen Überblick über die europäische Literatur andeutet, sondern auch als Inspiration für das Theaterrepertoire dienen durfte. [10] Die in der Chronik der Mark Rosswald sowie im Nachlass Eduard Richters [2] vermittelten Informationen über die Rosswalder Büchersammlung weisen auf Publikationen aus den Bereichen wie Philosophie, Naturgeschichte, Physik, Mathematik, Rede- und Dichtkunst sowie Medizin und Geographie hin.[3] Neben den auf die Gebiete wie Geographie, Jura, Chemie, Alchemie,

\footnotetext{
${ }^{4}$ Auf diese Tatsache weist Milan Myška in seiner Monografie über Albert Joseph Hoditz hin. [10]

${ }^{5}$ Die Beschäftigung mit dem Katalog der Schlossbibliothek von Rosswald stützt sich auf die im Landesarchiv Troppau deponierte „Chronik der Markt Rosswald“ sowie auf den Nachlass des Regionalhistorikers Eduard Richter und auf die Erforschungen Milan Myškas.
} 
Gartenkunst ausgerichteten Publikationen wurden der Bibliothek literarische Werke der europäischen Autoren zugefügt.[3]

Das Repertoire des Schlosstheaters ist teilweise aus der literarischen Büchersammlung abzulesen, in der die Komödien, Tragödien und Possen von Molière (1622-1673), Pierre Corneille (1606-1684), François Marie Arouet Voltaire (1694-1778), Ludwig Holberg (16841754) etc. zu finden sind. Vom Katalog der Rosswalder Schlossbibliothek lassen sich jedoch auch deutschsprachige Dramen nicht trennen, unter denen die Werke Christian Fürchtegott Gellerts (1715-1769) und Gotthold Ephraim Lessings (1729-1781) dominieren. [10]

Für die Theater- und Musikaufführungen in Rosswald waren die Werke von Ignaz Pintus (1714-1786) bestimmt, die im Inventar der Schlossbibliothek vorlagen. [10] Die Prosawerke stammten von den Autoren der Antike ${ }^{6}$ und der Renaissance ${ }^{7}$ sowie von den Repräsentanten der europäischen Aufklärung. ${ }^{8}$

Informationen über die Rosswalder Schaubühne bietet nicht zuletzt der mit Großhoschütz verbundene Nachlass, in dem neben den Sentenzen des Grafen Hoditz das diesem Adligen dedizierte Vorspiel „Die Vereinigung des Trauerspiels, des Lustspiels, der Tonkunst und des Tanzes" von Johann Heinrich Friedrich Müller überliefert ist. ${ }^{9}$

Das Theatergeschehen in der Rosswalder Schlossresidenz ist ebenfalls vor dem Hintergrund der hiesigen Musikbühne zu betrachten, ${ }^{10}$ deren Tätigkeit mit dem Wirken Karl Ditters von Dittersdorfs, des italienischen Librettisten Ignaz Pintus, eines Freundes Ludwig van Beethovens, Karl Hanke ${ }^{11}$, des Schülers Christoph Willibald Glucks Heinrich Klein ${ }^{12}$ etc. verknüpft war. ${ }^{13}$ Neben den Werken der genannten Komponisten wurden in Rosswald die aufklärerischen Tragödien Voltairs „Zayre“",Alzire, ou les Americains“ [4] sowie Lessings „Miss Sara Sampson“ und George Lilos „George von Barnwel, oder der Kaufmann von London" sowie klassizistische Dramen Molières aufgeführt.

In die Geschichte des Rosswalder Schlosstheaters sind auch Aufführungen der dramatischen Werke des Grafen Albert Joseph Hoditz eingegangen. Er schrieb nicht nur die Libretti zu den Opern „Die vergötterte Sophia“ und „Einquartierung der Panduren“, sondern sein literarisches Schaffen schließt auch die Possen „Doktor und Apotheker“" und „Der theuere Ziegenbock“ ein. [10]

Neben dem Einblick des Grafen Albert Joseph Hoditz in die zeitgenössische europäische Literatur, der Ausbildung seiner Bedienten zu Schauspielern und Opernsängern [10] und seiner Zusammenarbeit mit einigen westschlesischen Schlossresidenzen wurde das Rosswalder kulturelle Leben durch die Tätigkeit des Dramatikers und Schauspielers Johann Heinrich Friedrich Müller (1738-1815) bereichert, [21] der sich u. a um die Einstudierung der

\footnotetext{
${ }^{6}$ In der Bibliothek waren z. B. die Werke von Vergils, Plutarchs, Ovids etc. zu finden. Vgl. [10]

${ }^{7}$ Die Epoche der Renaissance wird in der Bibliothek durch das literarische Schaffen Erasmus von Rotterdams repräsentiert. Vgl. [10]

${ }^{8}$ Vertreten waren die Romane Daniel Defoes. Vgl. [10]

${ }^{9} \mathrm{Zu}$ dieser Problematik schrieb die Autorin dieses Artikels 2019 eine Studie über Müllers dramatische Tätigkei im Rosswalder Schlosstheater. Vgl. [21]

${ }^{10}$ Milan Myška weist auf die Tätigkeit einiger Musiker im Rosswalder Schlosstheater sowohl unter Karl Joseph Hoditz als auch unter seinem Sohn, Albert Joseph Hoditz hin. Vgl. [10]

${ }^{11}$ Die Lebensdaten sind unbekannt. Sein überliefertes Werk „Der Wunsch mancher Mädchen“ ist jedoch 1781 erschienen.

${ }^{12}$ Die Lebensdaten sind unbekannt.

13 Einige dieser Persönlichkeiten der europäischen Musikgeschichte haben sich an der Prägung der musikalischen Szene in einigen westschlesischen Schlossresidenzen beteiligt.
} 
Dramen Gotthold Ephraim Lessings verdient machte. Eine prägende Persönlichkeit stellte im Rosswalder Theaterleben ebenfalls Johann Christoph Gottlieb ${ }^{14}$ dar, der nach 1761 mit der Leitung des Schlosstheaters beauftragt wurde. Dennoch wurden über sein Wirken in dieser Kulturstätte fast keine Informationen überliefert.

Das kulturelle Engagement des Grafen Albert Joseph Hoditz hat zur Bereicherung des westschlesischen künstlerischen Kolorits zwischen Barock und Aufklärung wesentlich beigetragen. Die kulturellen Ambitionen dieses Grafen wären jedoch ohne seine Zusammenarbeit mit dem Grafen Chorinsky und dem Bischof Schaffgotsch nicht denkbar, die mit den Schlössern Großhotschütz und Johannesberg verbunden sind.

\section{Die literatur- und kulturgeschichtliche Bedeutung der Großhoschützer Schlossresidenz im Fokus der Archivforschung}

Die zwischen den westschlesischen Schlossresidenzen bestehende „kulturelle Synergie“ fällt bereits bei der Sichtung des Großhoschützer Archivnachlasses auf, der sich im Landesarchiv Troppau befindet. In diesen Archivakten sind die auf der Rosswalder Theaterbühne aufgeführten Dramen ${ }^{15}$ sowie die Korrespondenz mit den Komponisten ${ }^{16}$ überliefert, die das kulturelle Leben in einigen westschlesischen Schössern mitgeprägt haben. Diese Tatsache wird in der Biographie Karl Ditters von Dittersdorfs (1739 - 1799) unterstrichen, in der die musikalische Zusammenarbeit dieses Komponisten mit dem Großhoschützer sowie Rosswalder Orchester zugunsten des Besuchs des preußischen Monarchen folgendermaßen skizziert wurde: ,[...] ich hatte die beiden kleinen Kapellen der Grafen Hoditz und Chorinsky ${ }^{17}$ zusammengeschmolzen. Beim Eintritt des Prinzen begann eine kleine Symphonie von mir, nach welcher ein Konzert spielte." [7] Darüber hinaus soll Karl Ditters von Dittersdorf für die Rosswalder Musikszene die Opera buffa „Pancracio ed Esabella“ komponiert haben, die 1775 auch im Breslauer Theater aufgeführt wurde. [5]

Das Schloss Großhoschütz wurde vor allem in den 60er und 70er Jahren des 18. Jh. zum Zentrum des kulturellen Lebens in der westschlesischen Region. Der Graf Dominik Ignaz Chorinsky (1729-1792) unterhielt sein eigenes Orchester und Theater. ${ }^{18} \mathrm{Zu}$ den führenden Persönlichkeiten im kulturellen Mosaik dieses Schlosses gehörten Joseph Puschmann (17381794) und Ignaz Pintus (1714 - 1786). Das im Landesarchiv Troppau befindliche Bücherverzeichnis der Großhoschützer Bibliothek gewährt einen Einblick dieses Grafen in einige Wissensgebiete ${ }^{19}$ und in die zeitgenössische auf Deutsch sowie Französisch ${ }^{20}$ verfasste $^{2}$ Literatur.

\footnotetext{
${ }^{14}$ Seine Lebensdaten sind unbekannt.

${ }^{15}$ Im Archivnachlass des Großherrschaftsguts Großhoschütz ist z. B. das 1758 im Rosswalder Schlosstheater aufgeführte Vorspiel Johann Heinrich Friedrich Müllers „Vereinigung des Trauerspiels, des Lustspiels, der Tonkunst und des Tanzes“" zu finden. Vgl. [3]

16 In dem mit dem Großherrschaftsgut Großhoschütz verbundenen Archivnachlass ist der aus dem Johannesberger Schloss abgeschickte Brief des italienischen Librettisten Ignaz Pintus an den Grafen Ignaz Dominik Chorinsky vom 6. Juli 1772 überliefert. Obwohl dieser Komponist im Schloss Großhoschütz tätig war, wurde durch sein Musikschaffen ebenfalls das kulturelle Leben in Rosswald sowie in Johannesberg geprägt. Seine musikalische Zusammenarbeit mit dem in Johannesberg tätigen Karl Ditters von Dittersdorf belegen die in der Staatsbibliothek Berlin überlieferten Opern „La Liberatrice Del Popolo Giudaico Nella Persia“"von 1773, „Hiob ein geistliches Singstück in zwo Abtheilungen“ von 1791 etc. Vgl. [24]

${ }^{17}$ Der Graf Dominik Ignaz Chorinsky (1729-1792) besaß das Schloss Großhoschütz und war kulturell tätig.

${ }^{18}$ Neben diesem Schloss haben sich die kulturellen Interessen dieses Adligen in seinem Palais in Troppau durchgesetzt.

${ }^{19}$ Es handelt sich vor allem um die historisch ausgerichteten Abhandlungen und Lexika. Mit der Zeitspanne 1772-1779 sind zahlreiche Zeitungen verbunden, die Informationen über das zeitpolitische Geschehen in
} 
Darüber hinaus weisen die im Landesarchiv Troppau befindlichen Archivakten auf das Großhoschützer Theaterleben hin. [3] Da die genannten westschlesischen Schlossresidenzen kulturell zusammengearbeitet haben, dürften sich die im Großhoschützer Nachlass überlieferten Informationen ebenfalls auf die Schlösser aus der Umgebung dieses Dominiums beziehen. In den Spielplan dieser Bühnen sind die Schauspiele Elias Franckes eingegangen [3], die stark didaktisch geprägt waren. Die Dramen waren vorwiegend durch die Verbindung des Musikalischen und Dramatischen gekennzeichnet und wurden durch eine Ballettvorstellung abgeschlossen.

Ins kulturelle Mosaik des Großhoschützer Adelshofs sind ebenfalls Dramen Gottlieb Stephanies (1741-1800) eingegangen [3], die auch nach dem Vorbild Calderon de la Barcas (1600-1681) frei erdichtet sind. ${ }^{21}$ Der Autor setzt sich in seinen Schauspielen mit den Fragen nach der menschlichen Stellung in der Welt und der Suche nach dem Schutz vor der Ungerechtigkeit auseinander. Dieses Drama wurde in den untersuchten schlesischen Dominien von der „Gesellschaft deutscher Schauspieler“ unter der Direktion von Karl von Morocz $^{22}$ vorgestellt. [3]

Das kulturelle Leben in Großhoschütz und in dessen Umgebung wird jedoch auch in einigen auf Lateinisch und Französisch verfassten Dramenentwürfen sowie in panegyrisch geprägten, lateinischen Poemen und Oratorien für die österreichische Kaiserin Maria Theresia und für den preußischen König Friedrich II skizziert. Im Großhoschützer Theater wurden meistens Opern italienischer und französischer Provenienz aufgeführt. Einige ihrer Libretti wurden von Ignaz Pintus niedergeschrieben. [3] Von der musikalischen Leitung des Schlossorchesters durch den späteren Olmützer Domkapellmeister Joseph Puschmann (1738-1794) zeugen nicht nur einige Opernaufführungen, sondern auch die im Landesarchiv Troppau befindliche „Kantate“ von 1777 belegt seine musikalische Tätigkeit in Westschlesien. [3]

\section{$5 \quad$ Das Schloss Johannesberg in Jauernig als kultureller Mittelpunkt Westschlesiens}

Obwohl das westschlesische kulturelle Kolorit in der zweiten Hälfte des 18. Jh. durch das gesellschaftlich-kulturelle Engagement des Grafen Dominik Chorinsky (1729-1792) markant geprägt wurde, bildete auch das Schloss Johannesberg in Jauernig einen kulturellen Mittelpunkt dieser Region.[14, 18] Die seit dem 14. Jh. im Besitz der Breslauer Bischöfe stehende Schlossresidenz erlebte besonders in der Zeitspanne 1766-1795 eine kulturelle Blütezeit.[20] Der in Johannesberg tätige Breslauer Bischof Gotthard Philipp Schaffgotsch verfügte über einen Einblick in die zeitgenössische Kunst sowie in ihre Geschichte, wie es auch die bis heute bestehende Bibliothek der Familie Schaffgotsch in Bad Warnbrunn ${ }^{23}$ andeutet. [9]

Europa boten und auf den gesellschaftspolitischen Überblick des Grafen Dominik Chorinsky hinweisen: Gazete de Viene von 1767 (3 Bde.), Leipziger Intelligenz von 1779 (1 Bd.), Brünner Intelligenz aus der Zeitspanne 1775 - 1776 (2 Bde.), Breslauer Zeitung von 1773 (1 Bd.), Gazette de Berlin von 1776 (1 Bd.), Hamburger Zeitung 1773 - 1776 (4 Bde.), Erlanger Zeitung von 1776 (1 Bd.), Erlanger Real Zeitung von 1777 - 1778 (2 Bde.) etc. Vgl. ZAO, FVH, Inventarnr.: 163, Kartonnr.: 93. Diese Tatsache hängt mit dem gesellschaftlichen Engagement des Grafen Chorinsky zusammen, der seit 1760 bis zu seinem Tode das Amt des Landeshauptmanns im preußischen Teil Schlesiens und seit 1760 in Österreichisch-Schlesien bekleidete.

${ }^{20}$ Im Verzeichnis sind z. B. die Werke Arnaud Berquins, François René de Chateaubriands etc. zu finden.

${ }^{21}$ Es handelte sich z. B. um das Drama „Der Oberamtmann und die Soldaten“.

${ }^{22}$ Die Lebensdaten sind unbekannt. Er war als Theaterdirektor ungefähr seit den 50er Jahren des 18. Jh. tätig.

${ }^{23}$ Cieplice Zdròj 
Durch die Einladung des Komponisten Karl Ditters nach Johannesberg, seine Förderung und seine Beauftragung mit der Leitung des Johannesberger Orchesters ${ }^{24}$ wurde nicht nur das westschlesische kulturelle Leben beeinflusst, sondern in Johannesberg entstand auch die komische Oper „Doktor und Apotheker“. [16, 23] Einen Einblick in das Johannesberger kulturelle Engagement Karl Ditters von Dittersdorfs sowie in seine Verlagssorgen in den 1790er Jahren bietet die im Landesarchiv Troppau überlieferte Korrespondenz dieses Komponisten.[20] Obwohl die Johannesberger Tätigkeit Dittersdorfs meistens mit Festivitäten in den westschlesischen Dominien verbunden wird, ist seine musikalische Tätigkeit ebenfalls im Dienste der kirchlichen Liturgie zu betrachten.[19] Durch diesen Beitrag für die Bereicherung der westschlesischen Kirchenmusikkultur ist Ditters von Dittersdorf in die Nähe des im Piaristenkloster Weißwasser musikalisch tätigen Antonín Brossman (1731-1789) gerückt.

Das Schloss Johannesberg in Jauernig stellte in der Zeit zwischen Barock und Aufklärung einen der kulturellen Mittelpunkte der Region, und die kulturellen Initiativen Dittersdorfs übten einen Einfluss auf die Schlossresidenzen aus der Umgebung aus. Dank der musikalischen Tätigkeit des genannten Komponisten hat sich ebenfalls das musikalische Leben in den Dominien Großhoschütz und Rosswald beträchtlich entwickelt. Dank den kulturellen Kontakten und Ambitionen des Bischofs Gotthard Philipp Schaffgotsch wurde das westschlesische kulturelle Mosaik um neue Impulse bereichert, die sich in der skizzierten kulturellen Entwicklung manifestierten.

\section{Schlussfolgerungen}

Schlesien ist im Bereich der deutschsprachigen Literatur besonders in der Barockzeit durch das literarische Schaffen der Autoren wie Martin Opitz, Adreas Gryphius, Daniel Casper von Lohenstein, Christian Hoffmann von Hoffmannswaldau sowie deren Epigonen in den Vordergrund getreten. Die sog. Erste und Zweite schlesische Dichterschule ist von der deutschsprachigen Literaturgeschichte untrennbar. Trotzdem sind in der Literatur- und Kulturgeschichte dieser multikulturellen Region zahlreiche weiße Flecken zu finden, zu denen zweifelsohne die vorgestellten westschlesischen Schlossresidenzen unter dem literatur- und kulturgeschichtlichen Aspekt gehören.

Obwohl die Lage dieser Dominien als peripher $\mathrm{zu}$ betrachten ist, zeugen vor allem die Repertoires der untersuchten Schlosstheater sowie der rege Gedankenaustausch zwischen Albert Joseph Hoditz, Dominik Ignaz Chorinsky und dem Bischof Schaffgotsch von einem außergewöhnlichen kulturellen Aufschwung. Eine Schlüsselrolle spielten dabei die Künstler wie Karl Ditters von Dittersdorf, Johann Heinrich Friedrich Müller sowie der Graf Hoditz, der selbst literarisch tätig war.

Das Repertoire der Schlosstheater in Rosswald, Großhoschütz sowie in Johannesberg war vorwiegend von den französischen und italienischen barocken Vorbildern geprägt. Die Theateraufführungen schlossen dabei sowohl Dramen, als auch Ballett- und Opernvorstellungen ein. Die kulturellen Initiativen von Chorinsky, Hoditz und Schaffgotsch spielten sich oft jedoch immer noch im Dienste der barocken Repräsentation ab. Mit neuen Impulsen wurden die westschlesischen Kunststätten dank dem aufklärerisch orientierten kulturellen Engagement Johann Heinrich Friedrich Müllers konfrontiert, der auf diese Schlossbühnen die Dramen Gotthold Ephraim Lessings gebracht hat.

\footnotetext{
24 Mit dem musikalischen Schaffen Karl Ditters von Dittersdorf beschäftigten sich bereits zahlreiche Musikforscher. Einen Einblick in den mit dieser Thematik verknüpften Forschungsstand im Bereich der tschechischen Musikwissenschaft bietet der Artikel Rudolf Pečmans „,Tschechische Musikforscher über Carl Ditters von Dittersdorf“. Vgl. [11]
} 
Die kulturelle Präsenz auf den genannten westschlesischen Schlossresidenzen vervollständigt nicht nur ihr vielschichtiges kulturelles Bild in der Barockzeit, sondern wurde auch durch die repräsentativen Werke der Aufklärung gekennzeichnet.

\section{Literatur}

[1] Zemský archiv v Opavě (Landesarchiv Troppau), Velkostatek Slezské Rudoltice (Großherrschaftsgut Rosswald), Inventarnr.: 10452, Sig.: A 26.

[2] Pozůstalost Eduarda Richtera (Nachlass Eduard Richters), Zemský archiv v Opavě (Landesarchiv Troppau), Inventarnr.: 6.

[3] Zemský archiv v Opavě, Großherrschaftsgut Groß Hoschütz, Inventarnr.: 163, Kartonnr.: 93.

[4] MÜLLER, Johann Heinrich Friedrich: Vereinigung des Trauerspiels, des Lustspiels, der Tonkunst und des Tanzes. Troppau 1760. ZAO, FVH, Inventarnr.: 163, Kartonnr.: 93.

[5] ADRIÁNYI, Gabriel: Deutsche Musik in Ost- und Südeuropa. In: Studien zum Deutschtum im Osten. Nr. 28. Köln am Rhein - Weimar - Wien 1997, S. 7.

[6] BEIN, Werner: „,Der mährische Epikuräer“. Albert Joseph von Hoditz (1706-1778) als Mäzen der schönen Künste. In: SCHLAGER, Karlheinz (Hg.): Festschrift Hubert Unverricht. Tutzing 1992, S. 35.

[7] DITTERS VON DITTERSDORF, Karl: Lebensbeschreibung seinem Sohne in die Feder diktiert. Regensburg 1940.

[8] DRECHSLER, Paul: Albert von Hoditz. Ein Wundergraf von Rosswald. Ein Lebensbild. Leobschütz 1895.

[9] LABOREWICZ, Ivo: Die Bibliothek der Familie Schaffgotsch in Bad Warmbrunn. In: Silesia Nova. 5. Jg, Dresden-Wroclaw 2008, S. 75-78.

[10] MYŠKA, Milan: Hrabě Hodic a jeho svět. Zámecká kultura ve Slezsku mezi barokem a osvícenstvím. Ostrava 2011.

[11] PEČMAN, Rudolf: Tschechische Musikforscher über Carl Ditters von Dittersdorf. In: Unverricht, Hubert (Hg.): Carl Ditters von Dittersdorf. Leben - Umwelt - Werk. Tutzing 1997, S. 15-21.

[12] RACEK, Jiří: Z hudební minulosti zámku Hradce u Opavy. Př́íspěvek k hudebním dějinám Slezska. In: Časopis Slezského muzea. B, Nr. 22. Opava 1973, S. 1-21.

[13] RICHTER, Johannes: Der Wundergraf. Ein närrisches Genie - ein genialer Narr. Berlin 1937.

[14] RUCKOVÁ (ZLÁ), Iveta: Das Schloss Johannesberg in Jauernig im Spiegel der Literatur und Musik der deutschsprachigen Länder. In: Germanistik an tschechischen Universitäten. Gegenwart und Zukunft. Hradec Králové - Ostrava 2007, S. 191-196.

[15] SONDERMANN, Frieder: Notizen über einige Hunde-Epitaphe des Grafen Hoditz (1706-1778). In: Zeitschrift für deutsche Philologie. Nr. 115. Berlin 1996, S. 18.

[16] TARLINSKI, Piotr: Musikgeschichte Schlesiens und die Schlesier. Ein zusammenfassender Überblick. In: Tarlinski, Piotr (Hg.): Hubert Unverricht. De Musica in Silesia. Opole 2007, S. 36 f. 
[17] TRAMPLER, Richard: Der Sonderling Hoditz in Rosswald. Eine historische Skizze. In: Österreichisch-schlesisches Vereinskalender für das Jahr 1877. Jg. 2. Wien 1876, S. 38-74.

[18] ULBRECHT, Siegfried: Johannesberg, Jauernig und Umgebung. Begegnungen von Personen und Kulturen in einer europäischen Region. Freiburg-Görlitz 2012.

[19] TARLINSKI, Piotr: Dittersdorfs Kirchenmusik in Schlesien. Theologische Anmerkungen. In: TARLINSKI, Piotr - UNVERRICHT, Hubert: Karl Ditters von Dittersdorf. Beiträge zu seinem Leben und Werk. Opole 2000, S. 207-229.

[20] UNVERRICHT, Hubert: Carl Ditters von Dittersdorf. Leben - Umwelt - Werk. Tutzing 1997, S. 15-21.

[21] ZLÁ, I. Johann Heinrich Friedrich Müllers (1778 - 1815) Rosswalder dramatisches Intermezzo. In: Germanoslavica. Zeitschrift für germano-slawische Studien. 2019, Nr. 30(2).

[22] ZLÁ, I. Lebensweise des Grafen Albert Joseph Hoditz im Spiegel seines literarischen Schaffens. In: Brünner Beiträge zur Germanistik und Nordistik. 2018, Nr. 32(2), S. 8187.

[23] ZUBER, Rudolf. Karel Ditters z Dittersdorfu. Javorník 1999.

[24] STAATSBIBLIOTHEK ZU BERLIN: http://digital-beta.staatsbibliothek-berlin.de

doc. Mgr. Iveta Zlá, Ph.D. 


\section{VYBRANÉ ZÁPADOSLEZSKÉ ZÁMECKÉ REZIDENCE JAKO REPREZENTATIVNÍ SOUČÁST KULTURY MEZI BAROKEM A OSVÍCENSTVÍM. NÁRYS PROBLEMATIKY Z LITERÁRNĚ A KULTURNĚHISTORICKÉHO POHLEDU}

Středobodem studie je nastínění kulturního obrazu zámeckých sídel ve Slezských Rudolticích, Velkých Hošticích a na zámku Jánský Vrch. Pozornost je věnována nejen kulturním aktivitám Alberta Josefa Hodice, Dominika Skrbenského a Filipa Gottharda Schaffgotsche, ale také vzájemné kulturní provázaností zmíněných panství. Ústřední osobností kulturního života na uvedených zámcích se stal především Karel Ditters z Dittersdorfu, jehož hudební tvorbou byla doprovázená představení jak v zámeckých divadlech ve Slezských Rudolticích a ve Velkých Hošticích, tak na zámku Jánský Vrch. I když se kulturní život v západním Slezsku rozvíjel ve stínu sedmileté války, došlo $\mathrm{k}$ jeho výraznému rozmachu. V literárním a kulturním panoramatu západoslezských zámeckých rezidencí se odrážely nejen impulzy barokní kultury, ale byly také prrejímány podněty pramenící z osvícenské literatury, filozofie a životního stylu.

\section{Selected West Silesian Castle Residences as Cultural Representatives BETWEEN BAROQUE AND ENLIGHTENMENT. SUMMARY OF THE PROBLEMATICS IN THE LITERATURE AND CULTURAL VIEW}

The center of the study is an outline of the cultural image of the chateau residences in Slezské Rudoltice, Velké Hoštice and Jánský Vrch. Attention is paid not only to the cultural activities of Albert Josef Hodice, Dominik Skrbenský and Filip Gotthard Schaffgotsch, but also to the mutual cultural interconnectedness of the mentioned estates. Karel Ditters von Dittersdorf in particular became the central figure in the cultural life of the above-mentioned castles, whose musical work was accompanied by performances in the castle theaters in Slezské Rudoltice and Velké Hoštice and at castle Jánský Vrch. Although cultural life in western Silesia developed in the shadow of the Seven Years' War, it flourished significantly. The literary and cultural panorama of the chateau in Westsilesia reflected not only the impulses of Baroque culture, but also received stimuli from literature, philosophy and lifestyle of Enlightenment.

\section{WYBRANE REZYDENCJE ZAMKOWE ZACHODNIEGO ŚLĄSKA I ICH ROLA W KULTURZE BAROKU A OŚWIETLENIA. BADANIE Z LITERACKIEJ I KULTURALNEJ PERSPEKTYWY}

Centralnym punktem opracowania jest zarys kulturowego obrazu rezydencji zamkowych w Slezskich Rudolticach, Velkich Hošticach i w Jánskim vrchu. Zwraca się uwagę nie tylko na działalność kulturalną Alberta Josefa Hodica, Dominika Skrbenskiego i Filipa Gottharda Schaffgotscha, ale także na wzajemne powiązania kulturowe wspomnianych osiedli. Szczególnie Karel Ditters z Dittersdorfu stał się centralną postacią w życiu kulturalnym wyżej wymienionych zamków, którego twórczości muzycznej towarzyszyły występy zarówno w teatrach zamkowych w Slezskich Rudolticach i Velkich Hošticach i na zamku Jánský vrch. Życie kulturalne na Śląsku Zachodnim rozwijało się wprawdzie w cieniu wojny siedmioletniej, ale znacznie się rozwinęło. Literacka i kulturowa panorama zachodniośląskich rezydencji zamkowych odzwierciedlała nie tylko impulsy kultury barokowej, ale czerpała także bodźce z oświeceniowej literatury, filozofii i stylu życia. 